\title{
Uso de elementos parasitas em antenas monopolo planar para sistemas UWB.
}

\author{
Tiago C. Martins, Rodrigo M.S. de Oliveira e Carlos L.S.S. Sobrinho.
}

\begin{abstract}
Resumo - Este trabalho apresenta uma proposta de antena monopolo planar operando na faixa para sistemas de comunicação de banda ultralarga (UWB) de 3.1 a 10.6 GHz. Inicialmente, é analisada uma antena monopolo planar constituída por um condutor retangular de largura $w=12 \mathrm{~mm}$ e altura $\mathrm{h}=20 \mathrm{~mm}$ sobre um plano de terra, a qual tem largura de banda de $3,5 \mathrm{GHz}$. Aqui, foram plotadas: a curva de perda de retorno e o diagrama de irradiação, os quais estão de acordo com os resultados da literatura. Foi projetada uma outra antena monopolo planar com altura $h=14 \mathrm{~mm}$ e largura $w=8 \mathrm{~mm}$, a qual possui largura de banda igual a $6,2 \mathrm{GHz}$. Em seguida, foram utilizados três elementos parasitas em conjunto com a antena anterior, sendo obtida uma antena de largura de banda de $6,5 \mathrm{GHz}$ dentro da faixa de 3.1 a $10.6 \mathrm{GHz}$. As freqüências de corte superior e inferior, utilizadas para calcular a largura de banda, foram obtidas em -10 dB. Todas as análises foram realizadas a partir de simulações utilizando o método FDTD.
\end{abstract}

Palavras-Chave - Elementos parasitas, Antena Monopolo Planar, Método FDTD, sistemas UWB.

\section{I.INTRODUÇÃO}

Atualmente, as antenas de banda ultralarga (UWB) têm atraído a atenção de pesquisadores do mundo todo face à grande importância desse tipo de antena em atender as exigências dos sistemas de comunicações sem fio operando na faixa de 3,1 a 10,6 GHz. Desta forma, vários tipos de antenas têm sido propostos, tais como: as antenas logperiódica, corneta, monocone, Vivaldi, fractal, monopolo, etc [1-4]. Dentre essas antenas, o monopolo, devido as suas características atrativas (como operar em uma ampla faixa de freqüências, diagrama de irradiação quase onidirecional, estrutura simples e de baixo custo) merece atenção [5-9]. Neste trabalho são propostas algumas técnicas com a finalidade de aumentar a largura de banda de uma antena monopolo planar retangular e sintonizá-la.

\section{II.O MÉtodo FDTD}

\section{A. Teoria}

O método das diferenças finitas no domínio do tempo ou método FDTD aproxima as derivadas de equações diferenciais por diferenças finitas. Em problemas de propagação de ondas eletromagnéticas, as equações diferenciais utilizadas são as equações de Maxwell, mais especificamente, as leis de Faraday (eq. (1)) e de Ampère (eq. (2)), para um meio linear, isotrópico e com perdas:

$$
\nabla \times \vec{E}=-\mu \frac{\partial \vec{H}}{\partial t}
$$

-Universidade Federal do Pará, Departamento de Engenharia Elétrica e de Computação - tiago@lane.ufpa.br, rodrigo@lane.ufpa.br, leonidas@ufpa.br. e

$$
\nabla \times \vec{H}=\epsilon \frac{\partial \vec{E}}{\partial t}+\sigma \vec{E}
$$

nas quais $\vec{E}$ é o vetor intensidade de campo elétrico $(\mathrm{V} / \mathrm{m})$, $\vec{H}$ é o vetor intensidade de campo magnético $(\mathrm{A} / \mathrm{m}), \mu, \epsilon \mathrm{e}$ $\sigma$ são os parâmetros constitutivos do meio, respectivamente: permeabilidade magnética, permissividade elétrica e condutividade elétrica.

A célula de Yee [10], mostrada na Figura 1, permite a elaboração de um algoritmo eficiente para implementações utilizando o método FDTD. É possível fazer a distribuição espacial discreta dos campos, a qual implica também em uma discretização no tempo. Porém, a fim de evitar a dispersão numérica e a instabilidade do método, é necessário atender às seguintes condições [11]:

$$
\Delta x, \Delta y, \Delta z \leq \frac{\lambda_{\min }}{10}
$$

$\mathrm{e}$

$$
\Delta t \leq \frac{1}{v_{\max } \sqrt{\frac{1}{\Delta x^{2}}+\frac{1}{\Delta y^{2}}+\frac{1}{\Delta z^{2}}}}
$$

nas quais $\lambda_{\min }$ é o menor comprimento de onda analisado; $\Delta x, \Delta y$ e $\Delta z$ são os incrementos espaciais correspondentes às dimensões da célula e $\Delta t$ é o incremento temporal, onde a equação (4) é conhecida como condição de Courant.

\section{B. UPML}

Como neste trabalho é estudada a propagação de ondas eletromagnéticas em um ambiente aberto, faz-se necessário o uso de uma técnica de truncagem do domínio de análise, que funcione como uma câmara anecóica virtual, absorvendo toda radiação que incida sobre as fronteiras da região analisada. Dessa forma, são simuladas ondas eletromagnéticas propagando-se para o infinito sem que ocorram reflexões, as quais comprometeriam os resultados obtidos. Essa técnica de absorção é baseada no uso de camadas perfeitamente casadas com anisotropia uniaxial ou UPML (Uniaxial Perfectlly Matched Layers) [12]. A seguir são mostradas, para um meio com anisotropia uniaxial, as equações de atualização das componentes $\mathrm{x}$ dos vetores densidade de fluxo elétrico $\left(\mathrm{D}_{\mathrm{x}}\right)$ e intensidade de campo elétrico $\left(\mathrm{E}_{\mathrm{x}}\right)$, assim como, as equações de atualização das componentes $\mathrm{z}$ dos vetores densidade de 
fluxo magnético $\left(\mathrm{B}_{\mathrm{z}}\right)$ e intensidade de campo magnético $\left(\mathrm{H}_{\mathrm{z}}\right)$. Essas equações foram usadas para as camadas absorventes e para o domínio de análise, sendo que para este, os parametros $\sigma$ são iguais a zero.

$$
\begin{aligned}
& D_{x\left(i+\frac{1}{2}, j, k\right)}^{n+1}=D_{x\left(i+\frac{1}{2}, j, k\right)}^{n}\left(\frac{1-\frac{\sigma_{z} \Delta t}{2 \epsilon_{0}}}{1+\frac{\sigma_{z} \Delta t}{2 \epsilon_{0}}}\right)+\left(\frac{\Delta t}{1+\frac{\sigma_{z} \Delta t}{2 \epsilon_{0}}}\right) \times \\
& {\left[\frac{H_{z\left(i+\frac{1}{2}, j+\frac{1}{2}, k\right)}^{n+\frac{1}{2}}-H_{z\left(i+\frac{1}{2}, j-\frac{1}{2}, k\right)}^{n+\frac{1}{2}}}{\Delta y}-\frac{H_{y\left(i+\frac{1}{2}, j, k+\frac{1}{2}\right)}^{n+\frac{1}{2}}-H_{y\left(i+\frac{1}{2}, j, k-\frac{1}{2}\right)}^{n+\frac{1}{2}}}{\Delta z}\right],} \\
& E_{x\left(i+\frac{1}{2}, j, k\right)}^{n+1}=E_{x\left(i+\frac{1}{2}, j, k\right)}^{n}\left(\frac{1-\frac{\sigma_{y} \Delta t}{2 \epsilon_{0}}}{1+\frac{\sigma_{y} \Delta t}{2 \epsilon_{0}}}\right)+\frac{1}{\epsilon_{0} \epsilon_{r}\left(1+\frac{\sigma_{y} \Delta t}{2 \epsilon_{0}}\right)} \times \\
& {\left[D_{x\left(i+\frac{1}{2}, j, k\right)}^{n+1}\left(1+\frac{\sigma_{X} \Delta t}{2 \epsilon_{0}}\right)-D_{x\left(i+\frac{1}{2}, j, k\right)}^{n}\left(1-\frac{\sigma_{X} \Delta t}{2 \epsilon_{0}}\right)\right] \text {, }} \\
& B_{z\left(i+\frac{1}{2}, j+\frac{1}{2}, k\right)}^{n+\frac{1}{2}}=B_{z\left(i+\frac{1}{2}, j+\frac{1}{2}, k\right)}^{n-\frac{1}{2}}\left(\frac{1-\frac{\sigma_{y} \Delta t}{2 \epsilon_{0}}}{1+\frac{\sigma_{y} \Delta t}{2 \epsilon_{0}}}\right)+\left(\frac{\Delta t}{1+\frac{\sigma_{y} \Delta t}{2 \epsilon_{0}}}\right) \times \\
& {\left[\frac{E_{x\left(i+\frac{1}{2}, j+1, k\right)}^{n}-E_{x\left(i+\frac{1}{2}, j, k\right)}^{n}}{\Delta y}-\frac{E_{y\left(i+1, j+\frac{1}{2}, k\right)}^{n}-E_{y\left(i, j+\frac{1}{2}, k\right)}^{n}}{\Delta x}\right]}
\end{aligned}
$$

e

$$
\begin{gathered}
H_{z\left(i+\frac{1}{2}, j+\frac{1}{2}, k\right)}^{n+\frac{1}{2}}=H_{z\left(i+\frac{1}{2}, j+\frac{1}{2}, k\right)}^{n-\frac{1}{2}}\left(\frac{1-\frac{\sigma_{x} \Delta t}{2 \epsilon_{0}}}{1+\frac{\sigma_{x} \Delta t}{2 \epsilon_{0}}}\right)+\frac{1}{\mu_{0}\left(1+\frac{\sigma_{X} \Delta t}{2 \epsilon_{0}}\right)} \times \\
{\left[B_{z\left(i+\frac{1}{2}, j+\frac{1}{2}, k\right)}^{n+\frac{1}{2}}\left(1+\frac{\sigma_{z} \Delta t}{2 \epsilon_{0}}\right)-B_{x\left(i+\frac{1}{2}, j+\frac{1}{2}, k\right)}^{n-\frac{1}{2}}\left(1-\frac{\sigma_{z} \Delta t}{2 \epsilon_{0}}\right)\right]}
\end{gathered}
$$

As demais componentes podem ser obtidas por uma troca cíclica dos índices envolvidos.

\section{Parâmetros para a simulação FDTD}

Os parâmetros de análise adotados para o método FDTD nas simulações foram: dimensões das células $\Delta x=\Delta y=$ $\Delta z=1,0 \mathrm{~mm}, 4000$ iterações temporais, excitação por um pulso gaussiano e UPML de 10 camadas como condição absorvente.

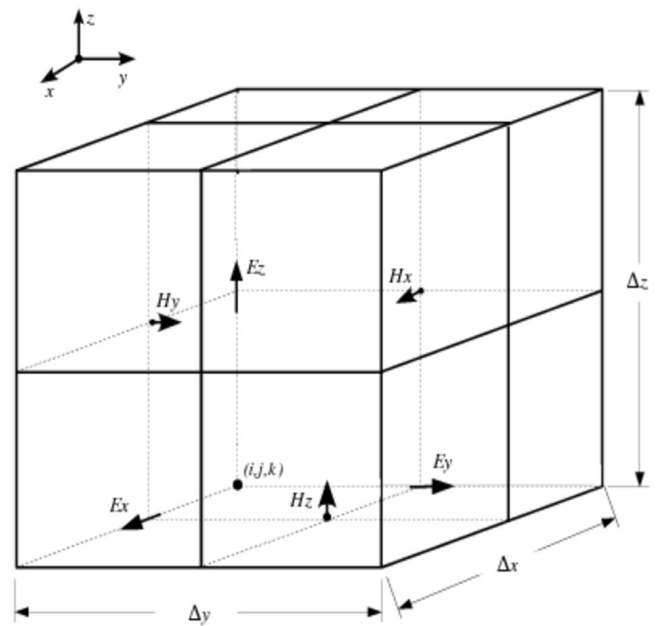

Fig. 1. A Célula de Yee e a distribuição espacial discreta das componentes dos campos elétrico e magnético.

\section{III.RESULTADOS}

\section{A. Monopolo Planar Simples}

A antena monopolo planar em análise é constituída por um condutor retangular de altura $\mathbf{h}=20 \mathrm{~mm}$ a partir do plano de terra e largura $\mathbf{w}=12 \mathrm{~mm}$, conforme mostrado na Figura 2, com uma distância de $1 \mathrm{~mm}$ entre o condutor e o plano de terra.

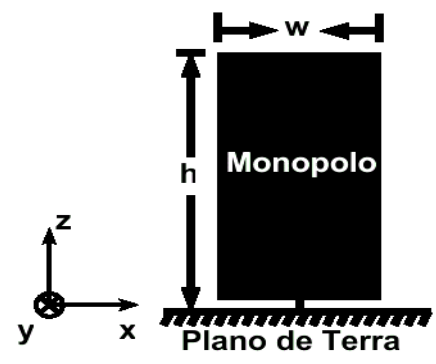

Fig. 2. Representação da antena monopolo planar de altura $\mathbf{h}$ e largura $\mathbf{w}$, no plano xz.

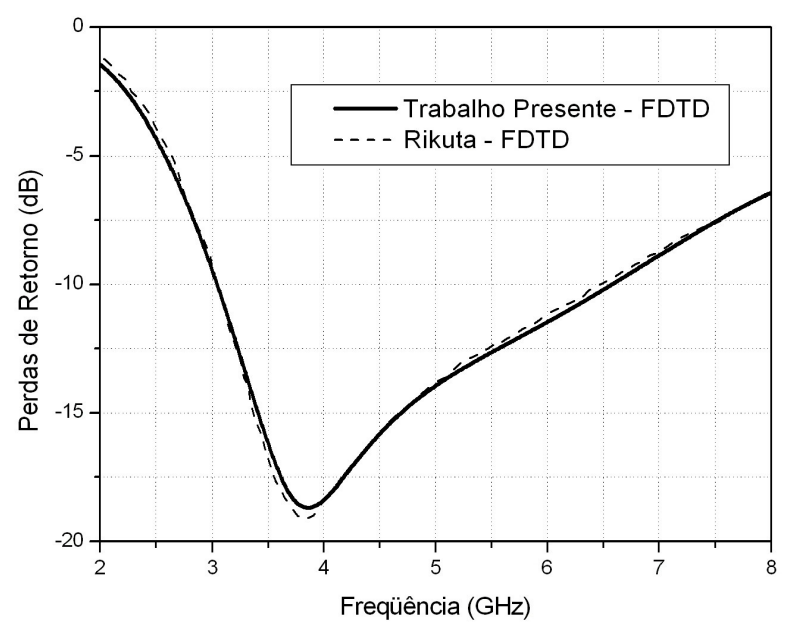

Fig. 3. Curva de Perdas de Retorno para a antena monopolo planar simples. 
Essa antena possui largura de banda de $3,5 \mathrm{GHz}$ (considerando perdas de retorno menores do que $-10 \mathrm{~dB}$ ). Foi admitido que a resistência da alimentação é de $50 \Omega$. Tanto a curva de perdas de retorno mostrada na Figura 3, quanto o diagrama de irradiação da antena, mostrado na Figura 4, estão de acordo com os resultados obtidos em [9].

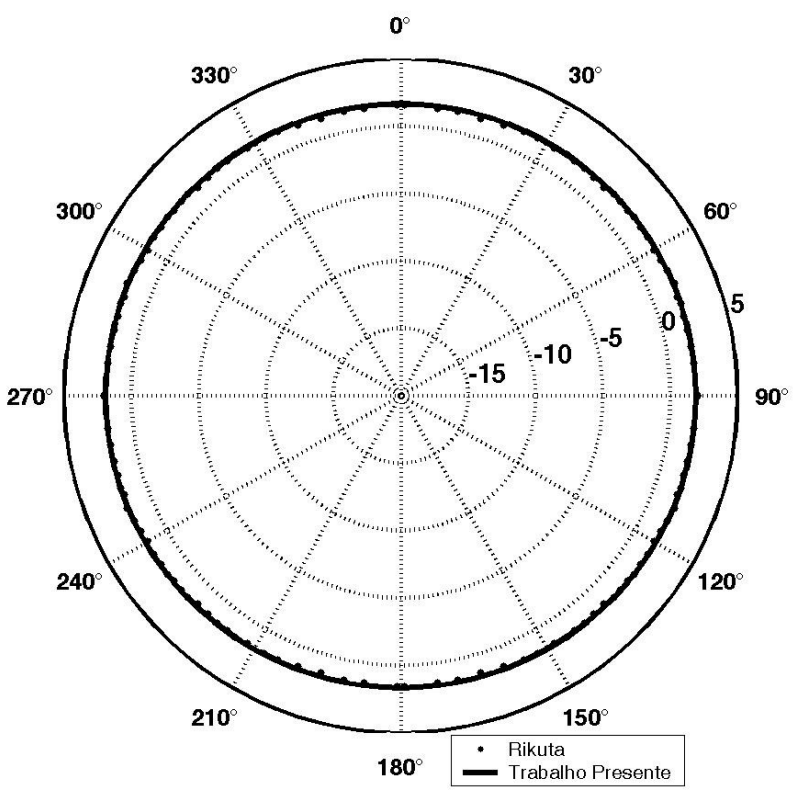

Fig. 4. Diagrama de irradiação da antena em dBi para a freqüência de $3 \mathrm{GHz}$, no plano $x y$.

\section{B. Elementos Parasitas}

Inicialmente foi simulada uma outra antena com dimensões otimizadas para a obtenção de uma maior largura de banda. As dimensões encontradas foram $\mathbf{h}=14 \mathrm{~mm}$ e $\mathbf{w}=8 \mathrm{~mm}$. A largura de banda obtida para essa antena foi de $6,2 \mathrm{GHz}$.

Em seguida, conjuntamente com essa antena, foram utilizados três elementos parasitas, de acordo com a configuração mostrada na Figura 5. Foram consideradas três configurações para os elementos parasitas: $\mathbf{a}=8, \mathbf{a}=6$ e $\mathbf{a}=$ 4. Nos três casos, foi utilizado $\mathbf{d}=1 \mathrm{~mm}$. Na Figura 6 , são comparadas as perdas de retorno. Como nos três casos analisados a faixa de operação das antenas excede o valor de 10,6 GHz, que é o limite de freqüência superior da faixa de interesse, foi admitido que a freqüência de corte superior é de $10,6 \mathrm{GHz}$, nos três casos. As freqüências de corte inferiores em -10 dB são $6,1 \mathrm{GHz}, 6,52 \mathrm{GHz}$ e $6,43 \mathrm{GHz}$, respectivamente, para $\mathbf{a}=8 \mathrm{~mm}, \mathbf{a}=6 \mathrm{~mm} \mathrm{e} \mathbf{a}=4 \mathrm{~mm}$. Conquanto, a maior largura de banda tenha sido obtida para a $=8 \mathrm{~mm}(6,61 \mathrm{GHz})$, o melhor resultado foi aquele obtido para a antena com $\mathbf{a}=6 \mathrm{~mm}$, pois, a mesma apresentou valores de perda de retorno consideravelmente menores do que aquele apresentado pela antena com $\mathbf{a}=8 \mathrm{~mm}$, tendo largura de banda apenas um pouco menor $(6,52 \mathrm{GHz})$.

A Figura 7 mostra a distribuição das componentes $\mathrm{z}$ do campo elétrico para uma antena com três elementos parasitas, para $\mathbf{h}=14 \mathrm{~mm}, \mathbf{w}=8 \mathrm{~mm}, \mathbf{a}=6 \mathrm{~mm} \mathrm{e} \mathbf{d}=1 \mathrm{~mm}$ na iteração de número 180 .

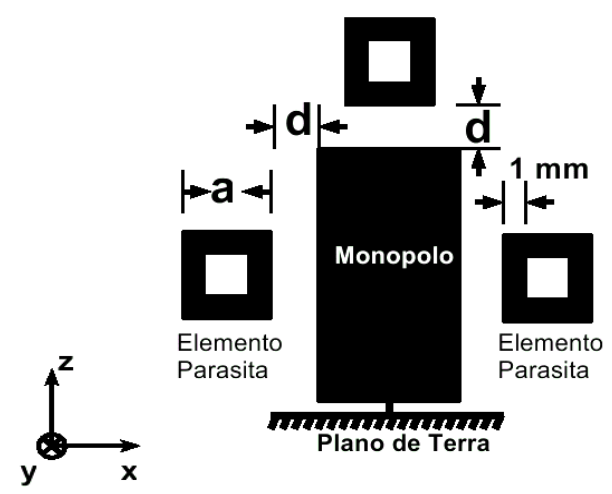

Fig. 5. Antena monopolo planar com três elementos parasitas quadrados de espessura $1 \mathrm{~mm}$, a um distância $\mathbf{d}=1 \mathrm{mmda}$ antena e de lado a.

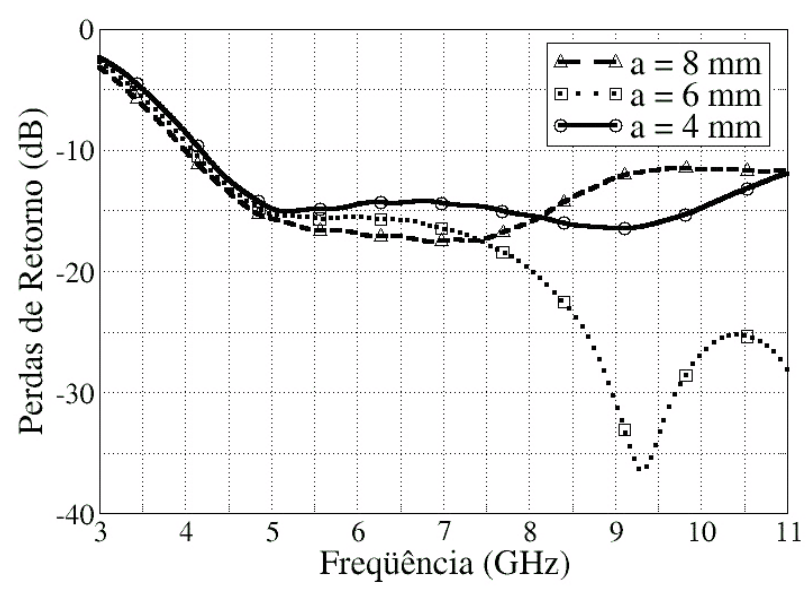

Fig. 6. Perdas de retorno $(\mathrm{dB})$ das antenas monopolo planar com três elementos parasitas quadrados de espessura $1 \mathrm{~mm}$, a um distância $\mathbf{d}=1 \mathrm{~mm}$ da antena e de lados $\mathbf{a}=8 \mathrm{~mm}, \mathbf{a}=6 \mathrm{mme} \mathbf{a}=4 \mathrm{~mm}$.

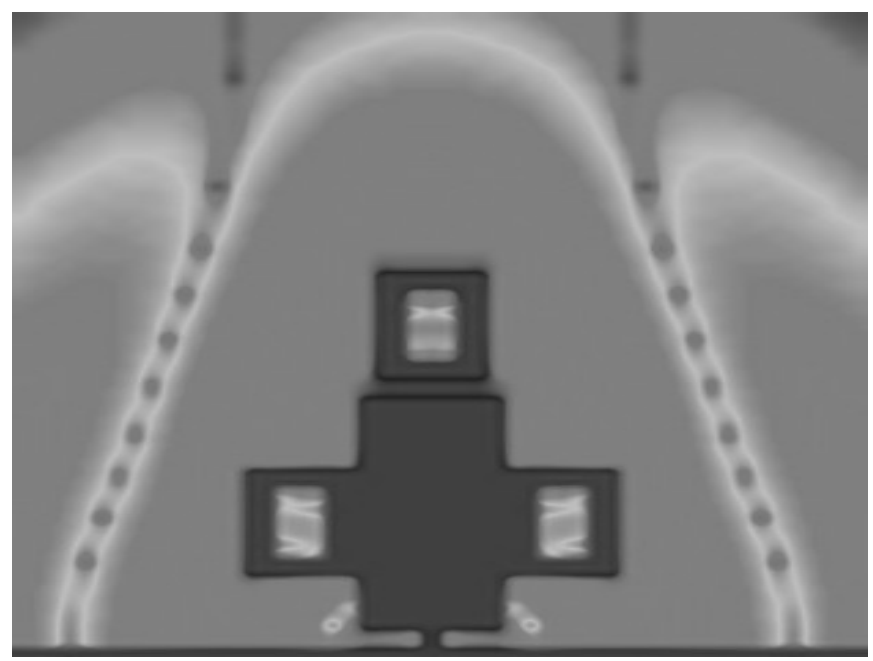

Fig. 7. Distribuição das componentes $\mathrm{z}$ do campo elétrico para uma antena com três elementos parasitas, para $\mathbf{h}=14 \mathrm{~mm}, \mathbf{w}=8 \mathrm{~mm}, \mathbf{a}=6 \mathrm{~mm} \mathrm{e} \mathbf{d}=1 \mathrm{~mm} \mathrm{na}$ iteração de número 180 
A Figura 8 mostra de forma detalhada os planos em que foram obtidos os diagramas de irradiação para a antena com três elementos parasitas, para $\mathbf{h}=14 \mathrm{~mm}, \mathbf{w}=8 \mathrm{~mm}, \mathbf{a}=6$ $\mathrm{mm}$ e $\mathbf{d}=1 \mathrm{~mm}$, nos planos $\mathrm{xy}, \mathrm{yz}$ e $\mathrm{xz}$, os quais são mostrados, respectivamente, nas Figuras 9, 10 e 11. A Figura 9 mostra que no plano xy o monopolo irradia de maneira uniforme em todas as direções. As Figuras 10 e 11 mostram que a antena praticamente não irradia na direção $\mathrm{z}$.

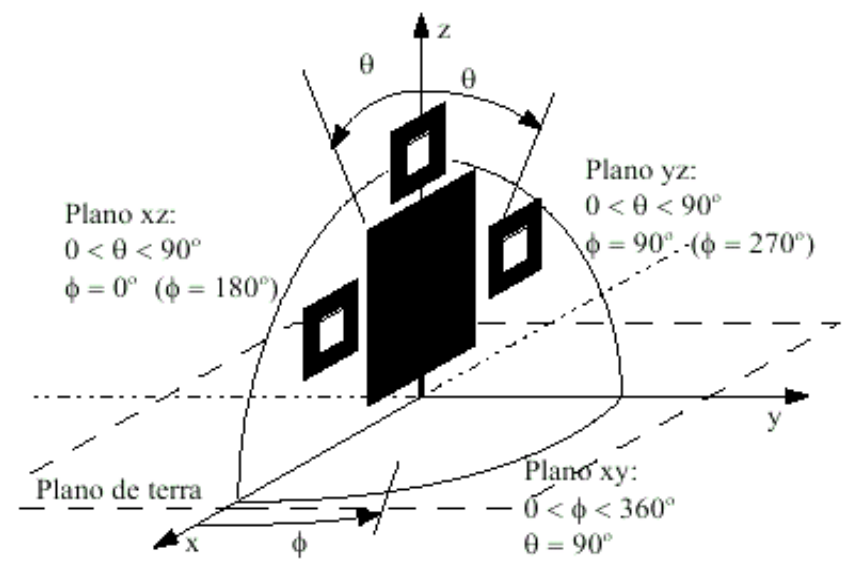

Fig. 8. Modelo de análise para obtenção dos diagramas de irradiação para a antena com três elementos parasitas, para $\mathbf{h}=14 \mathrm{~mm}, \mathbf{w}=8 \mathrm{~mm}, \mathbf{a}=6 \mathrm{~mm}$ e $\mathbf{d}=$ $1 \mathrm{~mm}$, nos planos xy, yz e xz.

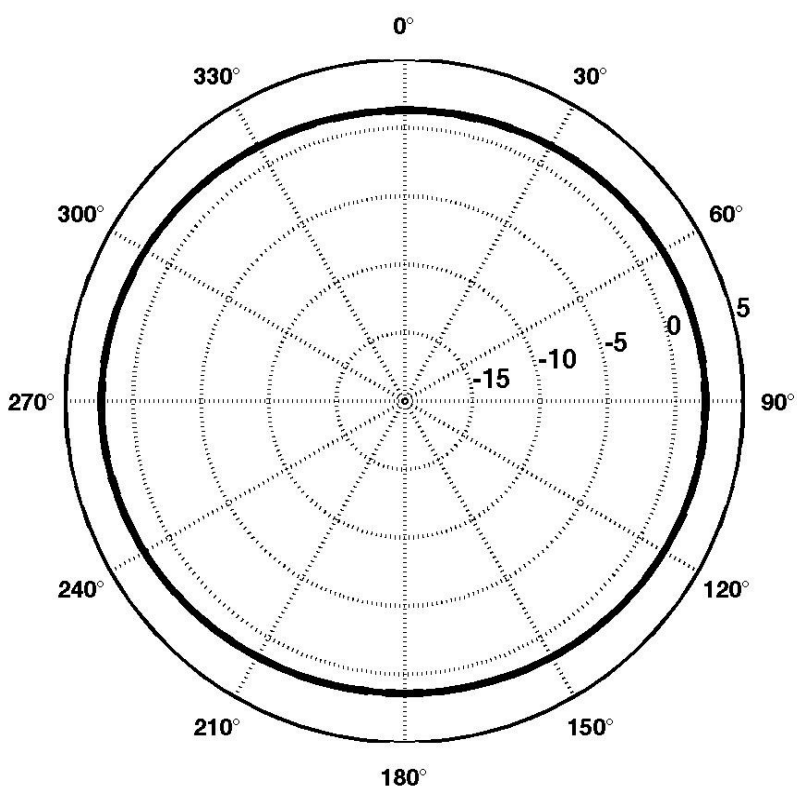

Fig. 9. Diagrama de irradiação para a antena com três elementos parasitas, para $\mathbf{h}=14 \mathrm{~mm}, \mathbf{w}=8 \mathrm{~mm}, \mathbf{a}=6 \mathrm{~mm}$ e $\mathbf{d}=1 \mathrm{~mm}$, no plano xy, na frequência de $6 \mathrm{GHz}$.

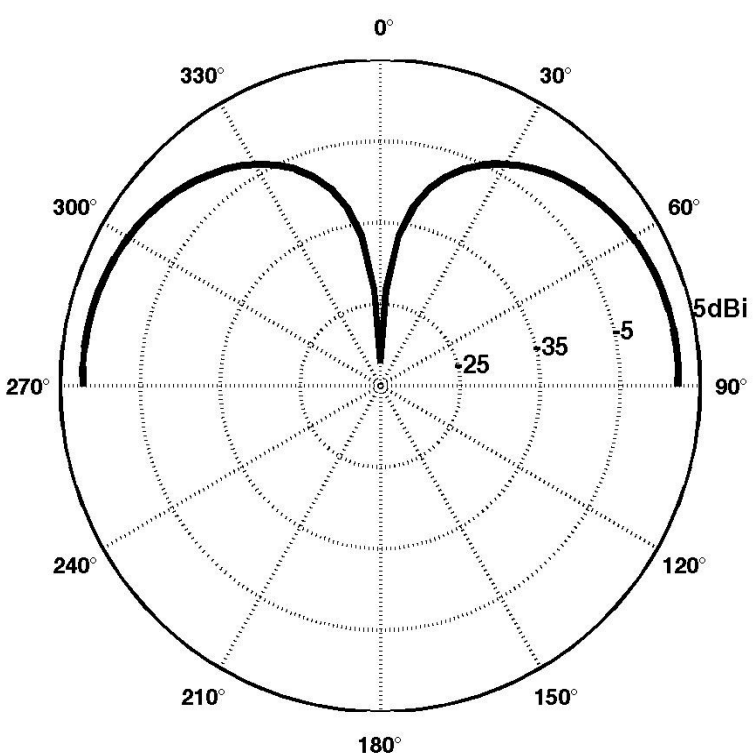

Fig. 10. Diagrama de irradiação para a antena com três elementos parasitas, para $\mathbf{h}=14 \mathrm{~mm}, \mathbf{w}=8 \mathrm{~mm}, \mathbf{a}=6 \mathrm{~mm}$ e $\mathbf{d}=1 \mathrm{~mm}$, no plano yz, na freqüência de 6 $\mathrm{GHz}$.

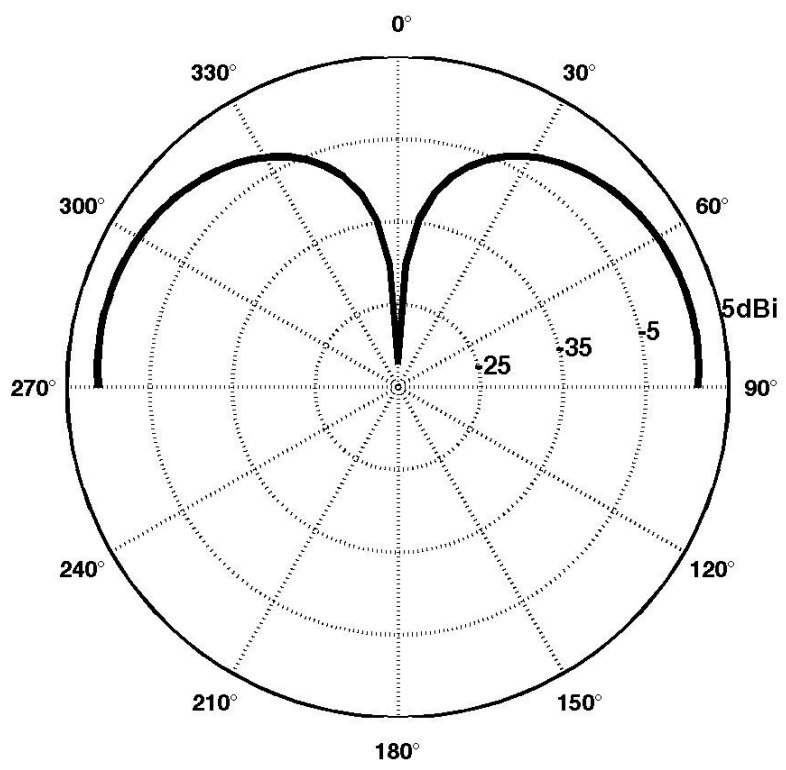

Fig. 11. Diagrama de irradiação para a antena com três elementos parasitas, para $\mathbf{h}=14 \mathrm{~mm}, \mathbf{w}=8 \mathrm{~mm}, \mathbf{a}=6 \mathrm{~mm}$ e $\mathbf{d}=1 \mathrm{~mm}$, no plano xz, na frequência de 6 $\mathrm{GHz}$.

\section{IV.ConCLusÃo}

Neste artigo foi proposta uma antena com três elementos parasitas, para $\mathbf{h}=14 \mathrm{~mm}, \mathbf{w}=8 \mathrm{~mm}, \mathbf{a}=6 \mathrm{~mm}$ e $\mathbf{d}=1 \mathrm{~mm}$, a qual apresenta um largura de banda de $6,52 \mathrm{GHz}(4,08$ a $10,6 \mathrm{GHz})$, dentro da faixa de $3,1 \mathrm{a} 10,6 \mathrm{GHz}$, utilizada para operação de sistemas de comunicação UWB. Foi mostrado 
que a antena sem os elementos parasitas apresentava largura de banda de 6,2 GHz. Dessa forma, vê-se que a técnica apresentada mostrou-se eficiente na realização do aumento de largura de banda de antenas monopolo planar. Para o desenvolvimento do projeto utilizou-se o método das diferenças finitas na solução numérica das equações rotacionais de Maxwell, onde a trucagem do domínio de análise foi feita através da técnica da UPML.

\section{Agradecimentos}

Este trabalho foi parcialmente custeado pelo $\mathrm{CNPq}$ e a Eletronorte.

\section{REFERÊNCIAS}

[1] T. Taniguchi and T. Kobayashi, "An Omnidirectional and Low-VSWR Antena for Ultra-Wideband Wireless Systems," 2002 Radio and Wireless Conf., pp. 145-148, Aug. 2002.

[2] N.P. AGRAWALL, G. Kumar and K.P. Ray, "Wide-Band Planar Monopole Antenna," IEEE Transactions Antennas Propagat., vol. 46, no 2, pp. 294-295, Feb. 1998.

[3] R.C. Comptopm, R.C. McPhedran, Z. Popovic, G.M. Rebeiz, P.P. Tong and D.B. Rutledge, "Bow-Tie Antennas on a Dielectric Half-Space: Theory and Experiment," IEEE Transactions Antennas Propagat., vol.35, no.6, pp. 622631, June 1987.

[4] M. Hammoud, P. Poey and F. colombel, "Matching The Input Impedance of A Broadband Disc Monopole," Electron. Lett., vol.29, no.4, pp. 406-407, Dec. 1993.

[5] Z.N. Chen, M.Y.W. Chia, and M.J. Ammann, "Optimization and comparison of Broadband monopoles", IEE Proc Microwave Antennas Propagat. 150 (2003), 429435.

[6] E. Antonino-Daviu, M. Cabelo-Fabrés, M. FerrandoBataller, and A. Valero-Nogueira, "Wideband double-fed planar monopole antennas", Electron lett. 39 (2003), 1635 1636.

[7] M.J. Ammann, Z.N. Chen, "Wideband monopole antennas for multiband wireless systems", IEEE Transactions Antennas Propagat. Mag. 45 (2003), 146-150.

[8] J. Liang, C.C. Chiau, X. Chen, and C.G. Parini, "Printed Circular Ring Monopole Antennas", Microwave and Optical Technology Letters, Vol.45, no.5, June 2005.

[9] Y. Rikuta and R. Kohno, "Planar Monopole Antenna with Dual Frequency for UWB System", 2003 IEEE Conference on Ultra Wideband Systems and Technologies, 176- 179.

[10] K. Yee, "Numerical solution of initial boundary value problems involving Maxwell's equations in isotropic media", IEEE Transactions on Antennas and Propagation, Vol.14, Issue 3, pp. 302-307, 1966.

[11] A. Taflove and S. Hagness, "Computational Electrodynamics: The Finite-Difference Time-Domain Method", 3rd ed, Artech House Publishers, ISBN 1-58053832-0, 2005.
[12] S.D. Gedney, "An anisotropic perfectly matched layer absorbing media for the truncation of FDTD latices", IEEE Transactions on Antennas and Propagation, Vol.44, pp.1630-1639, 1996. 